\title{
Clinical Outcome and Safety Profile of Patients Underwent Hair Transplantation Surgery by Follicular Unit Extraction
}

\author{
Rupak Bishwokarma Ghimire' \\ 'Department of Dermatology, Kathmandu Medical College, Sinamangal, Kathmandu, Nepal.
}

Introduction: Hair transplant surgery is a minimally invasive surgery, where hair follicle is transplanted from donor area of patient's own body to the desired area.

Methods: Patient with hair transplant surgery from a clinic were followed up for one year. The patients were asked for their complications within 2 days, 7 days, one month, 2 to 6 months and one year for assessment of results, side effects. At the end of one-year, subjective evaluation was done with patient's satisfaction to growth as- poor growth, satisfactory and good growth.

Results: Out of 152 patients, maximum patients 74 (48.6\%) were in age group 21-30, followed by 61 $(40.01 \%)$ in age group 31-40, mean age was 31.79, youngest being 21 years and oldest being 70 years of age. Among the complications, swelling of forehead was in more than two- thirds of the patients $106(69.74 \%)$, followed by temporary hair fall noted in $65(42.76 \%)$ of patients. Clients' remarks noted at the end on one-year revealed $86.18 \%$ as excellent results, $11.84 \%$ as satisfactory and $1.97 \%$ as poor results.

Conclusions: Hair transplant sugary by FUE is a relatively safe surgery with optimum results and minimum complications, which can be useful to patients with androgenetic alopecia.

Keywords: follicular unit extraction; hair transplantation; Nepal.

\section{INTRODUCTION}

Hair transplant surgery is a minimally invasive surgery, where hair follicle is transplanted from donor area of patient's own body to the desired area. Follicular Unit Extraction (FUE) is the modern technique where you extract single follicle units or grafts from in between and transplanted to the recipient site. ${ }^{1}$

Medical therapies can only help the miniaturized hairs to grow but cannot replace the hair in bald areas. Hair transplantation surgery can be a important method to restore the bald areas, which remains as a permanent solution. ${ }^{2}$ However, few very studies have been done in Nepal to assess the results and safety profile of hair transplantation surgery.

The objective of this study is to assess the clinical outcome; results and side effects with hair transplant

Correspondence: Dr. Rupak Bishwokarma Ghimire, Kathmand Medical College, Sinamangal, Kathmandu, Nepal. Email: rupakghi@ gmail.com, Phone: $+977-9802900007$. 
surgery by FUE in Nepal.

\section{METHODS}

This study is a descriptive cross-sectional study where patients were followed for one year, who underwent hair transplant surgery at Aavaran Skin Clinic, Battisputali, Kathmandu from $15^{\text {th }}$ July 2017 to $15^{\text {th }}$ February 2018 - a registered clinic for skin care and hair transplant surgery. Ethical clearance was taken from ERB of Nepal Health Research Council Reg No 113/2017.

Patients above 20 years of age, undergone hair transplant surgery for androgenetic alopecia were included in the study. Patients with age below 20 years, patients with indications other than androgenetic alopecia were excluded in the study. Patients with underlying bleeding disorders, uncontrolled hypertension and diabetes were also excluded during the surgery, considering the safety profile of the patients.

All cases completing one year of hair transplant surgery and meeting the inclusive criteria were included in the study. The patients were asked for their complications within 2 days, 7 days, one month, 2 to 6 months and one year for assessment of results, side effects. At the end of one-year, subjective evaluation was done with patient's satisfaction to growth as- poor growth, satisfactory and good growth. These responses were evaluated by phone calls and emails from the patients for those not attending the clinic.

The data were processed using an SPSS software package (SPSS 13.0 Inc., Chicago, IL, USA).

\section{RESULTS}

Total 152 patients were operated during the study period of 15 months. Out of 152 patients, 74 were in age group 21-30, 61 in age group 31-40, 14 in age group 41-50, 2 in age group 51-60 and 1 age group 61-70. Mean age was 31.79, youngest being 21 years and oldest being 70 years of age (Figure 1).

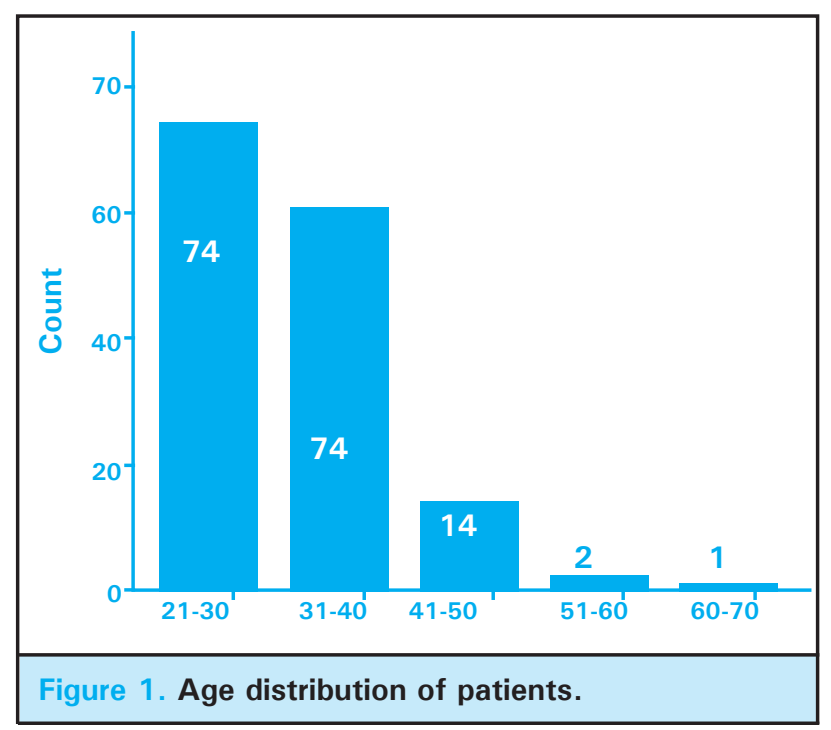

The number of grafts used for each patient were recorded and most patients $34.8 \%(n=53)$ had grafts filled between 2001 to $3000,26.9 \%(n=41)$ with grafts 3001 to $4000,19.07 \% \quad(n=29)$ with grafts 4001 to $5000,17.1 \%(n=26)$ with grafts 1000 to $2000,1.97 \%(n=2)$ with grafts 50001 to 6000 done by two sessions.

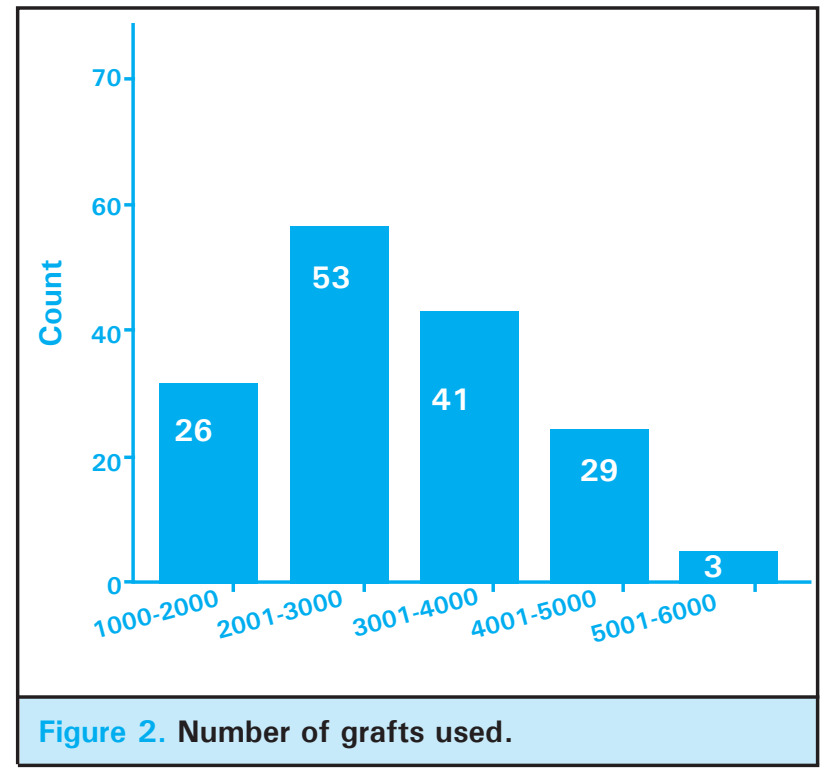

Peri operative complications were recorded with $60.53 \%$, out of which $34.87 \%$ had pain requiring diclofenac injection, $1.32 \%$ with increased blood pressure more than $140 / 90 \mathrm{~mm}$ of $\mathrm{Hg}$, hypotension with blood pressure less than $90 / 60 \mathrm{~mm}$ of $\mathrm{Hg}$ was recoded in $1.97 \%$, anxiety in $5.26 \%$, bleeding requiring tranexamic acid $500 \mathrm{mg}$ intramuscular in 1,97\%, Nausea in $9.21 \%$, Body ache in $3.95 \%$, Tachycardia (Pulse rate more than 100) in 0.66\%, Bradycardia with pulse less than 60 in $1.32 \%$. All complications with 
Ghimire. Is Clinical Outcome and Safety Profile of Patients Underwent Hair Transplantation Surgery by Follicular Unit Extraction

managed symptomatically.

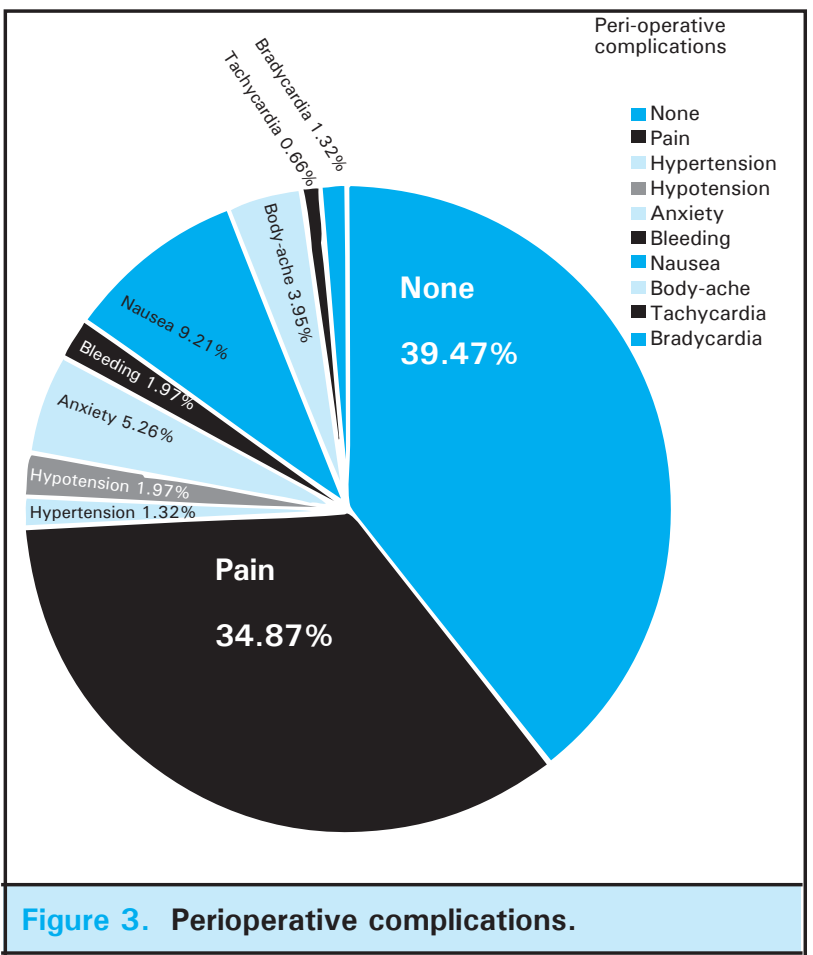

Immediate postoperative complications were noted from patients at follow up on two days while shampooing and removal of bandage. $71.68 \%$ developed complications, including swelling of forehead in $69.74 \%$, pain in $3.29 \%$, infection with yellowish discharge at recipient site in one patient $(0.66 \%)$.

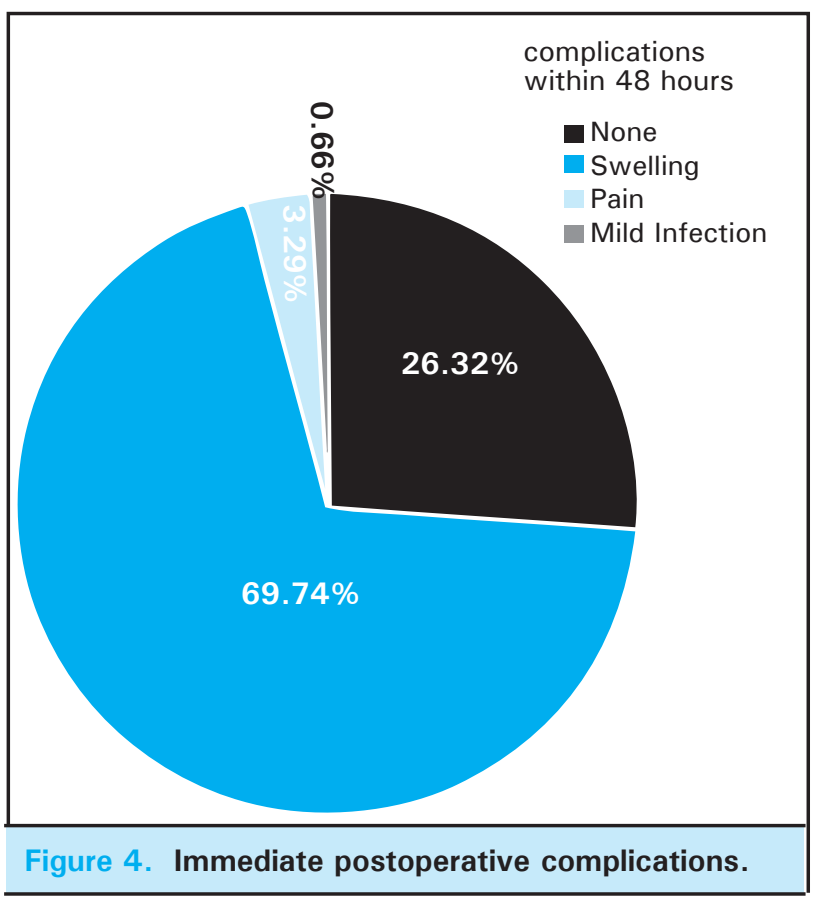

Late postoperative complications were evaluated at
7 days follow-up during removal of crusts which was noted in $30.94 \%$. Numbness of recipient site was seen in $9.87 \%$, Mild swelling was seen in $9.87 \%$, Itching at donor site in $4.61 \%$, Redness over recipient site in $3.95 \%$, Pain during crust removal in $1.97 \%$ and folliculitis at donor area in one patient $(0.66 \%)$.

Patients were again reviewed at 2 months, out of which $42.76 \%$ complained of hair fall, dryness in $5.26 \%$, itching in $4.61 \%$, folliculitis in $3.95 \%$, keloid in $1.97 \%$, numbness in $1.32 \%$.

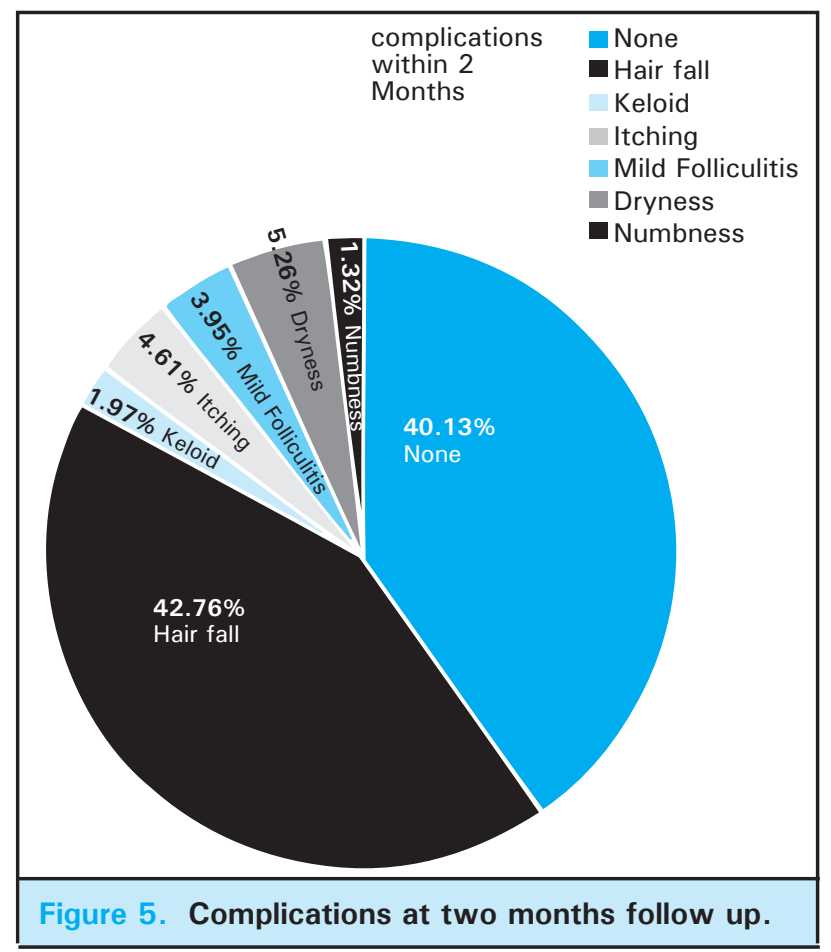

Patients were again called at six months completion, out of which only $9.87 \%$ patients had some complications or complaints in between. Among them, Folliculitis was noted in $1.97 \%$, Dandruff like scales in $1.97 \%$, Mild hair fall in $1.97 \%$, Scar formation in $1.32 \%$, Itching in $1.32 \%$ and Keloid formation in $0.66 \%$.

Patient remarks noted at the end on one year revealed $86.18 \%$ as good results, $11.84 \%$ as satisfactory and $1.97 \%$ as poor results.

\section{DISCUSSION}

In our study, out of 152 patients, 74 were in age group 21-30, 61 in age group 31-40, 14 in age group 41-50, 2 in age group 51-60 and 1 age group 61-70. Mean age was 31.79 , youngest being 21 years and oldest being 70 years of age. This finding was similar to the study done by Loganathan E et al in India, where the mean 
Ghimire. Is Clinical Outcome and Safety Profile of Patients Underwent Hair Transplantation Surgery by Follicular Unit Extraction

age was 30.06 years with the youngest one being 21 years and the oldest being 60 years. Postoperative edema was the most frequent complication, which was found in 31 (42.47\%) patients which was seen in $\mathrm{n}=106(69.74 \%)$ of our patients. ${ }^{3}$

No study has reported extensive follow up like this study, at two days post-operative, seven days, two months, six months and one year follow up. Barrerra A carried out a study among 100 patients from March 1994 to August 1996, by strip method with follow up of 5 months to 33 months, included 96 men and 4 women wose ages ranged from 21 to 67 years, with an average age of 42 years. Out of total 113 patients, 13 of them lost follow up. Complications encountered were hypertrophic scar at donor site in one patient $(1 \%)$, selfresolving ingrown hairs in nine patients $(9 \%)$. Ninety seven percent $(97 \%)$ patients were satisfied with results obtained after one session, which was similar to our patients, where $98.03 \%$ said they and good and satisfactory results. Three $(3 \%)$ patients who were dissatisfied with results after one session were pleased with results obtained after a second procedure. ${ }^{4}$

A retrospective study, in São Paulo, Brazil included data from 445 patients (mean age, $36.9 \pm 10.4$ yrs.) who underwent a total of 533 surgical hair transplantation procedures at the Plastic Surgery Division of Santa
Casa de Misericórdia de São Paulo or in a private clinic from February 1, 1995 to January 1, 2006. Most of the patients were men $(93.7 \%)$ with a mean age of 36.2 years \pm 9.7 years which was a little higher than our study. The overall complication rate was $4.7 \%$, including enlarged scar $(1.2 \%)$, folliculitis $(1.0 \%)$ necrosis in the donor area $(0.8 \%)$, keloids $(0.4 \%)$, bleeding $(0.2 \%)$, hiccups $(0.2 \%)$, infection $(0.2 \%)$, and pyogenic granuloma $(0.2 \%){ }^{5}$ However, the swelling of forehead, temporary hair loss have not been reported in this study.

The limitation of this study was the bias and confounding were not adjusted during this study. A more robust study design with long term follow up with appropriate statistical analysis would provide more closure look to the outcome of the hair transplant surgery.

\section{CONCLUSIONS}

Hair transplant sugary seems to be a relatively safe surgery with optimum results and minimum complications, which can be useful to patients with androgenetic alopecia. Larger multicenter studies can be done to further assess the results and complications.

Conflict of Interest: None.

\section{REFERENCES}

1. Dua Aman, Dua Kapil. Follicular Unit Extraction Hair Transplant. J Cutan Aesthet Surg. 2010 May-Aug; 3(2): 76-81. [DOI | Full Text]

2. Han SH, Byun JW, Lee WS, et al. Quality of life assessment in male patients with androgenetic alopecia: result of a prospective, multicenter study. Ann Dermatol. 2012;24(3):311-318. [PubMed]

3. Loganathan E, Sarvajnamurthy S, Gorur D, Suresh DH, Siddaraju MN, Narasimhan RT. Complications of hair restoration surgery: A retrospective analysis. Int J Trichol 2014;6:168-7. [PubMed | DOI]
4. Barrera A. Micrograft and minigraft megasession hair transplantation: review of 100 consecutive cases. Aesthet Surg J. 1997 May-Jun;17(3):165-9. [PubMed]

5. Salanitri S, MD; Gonçalves AJ, PhD; Helene A, Lopes FHJ. Surgical Complications in Hair Transplantation: A Series of 533 Procedures. Aesthetic Surg J. 2009; 29: 72-76. [PubMed] 